\title{
Experimental Study of User Selection for Dense Indoor Massive MIMO
}

\author{
Cheng-Ming Chen ${ }^{1}$, Qing Wang ${ }^{1}$, Abdo Gaber ${ }^{2}$, Andrea P. Guevara ${ }^{1}$, and Sofie Pollin ${ }^{1}$ \\ ${ }^{1}$ KU Leuven, Belgium $\quad{ }^{2}$ National Instruments Dresden, Germany \\ Email: \{cchen, qing.wang, aguevara, spollin\}@esat.kuleuven.be abdo.gaber@ni.com
}

\begin{abstract}
Multi-user massive MIMO is capable of serving at least ten users simultaneously. However, when users are closely located, their high inter-user correlation is undesired; under this condition, these densely packed users must be separated by higher layer scheduling. In this paper, a low complexity greedy user selection method combined with the incremental inter-userinterference minimization criterion is proposed. The resulting algorithm is evaluated using system level simulations that rely on the measured indoor line-of-sight channel, with 64 antennas in the base station at $2.61 \mathrm{GHz}$. Measurements are carried out using four different centralized and distributed base station antenna geometries, to evaluate the user selection performance for different indoor scenarios. Our evaluation shows that in a room with 64 densely deployed users, the proposed method increases the overall system sum rate, by up to $60 \%$. Moreover, when applying this method, the distributed deployment outperforms the collocated scenario by $18 \%$ on average.
\end{abstract}

\section{INTRODUCTION}

Massive MIMO was proposed in 2010 as a new paradigm to meet the increasing demand for wireless capacity [1]. It utilizes large arrays of antennas that span ten to hundreds of wavelengths in space, to serve more than ten users simultaneously. Using large number of antennas can significantly increase the network spectral efficiency [2].

\section{A. Motivation}

Traditional research on massive MIMO mainly focuses on the scenarios where user equipments (UEs) are sparsely distributed in an area. However, in many scenarios the UEs could be spatially collocated, and therefore, degrade the performance of traditional massive MIMO technologies because of the high inter-user-correlation (IUC). Within the same space, the UE density increases accordingly with the number of UEs. The uplink (UL) IUC under these scenarios are shown in Fig. 1. We can clearly see that with 64 UEs, the correlation is much higher than those with fewer number of UEs. For instance, more than $60 \%$ of the IUC is above 0.1 in the scenario with 64 UEs, while it is only $15 \%$ and $25 \%$ in the scenarios with 16 and 32 UEs, respectively. Note that with an IUC of 0.1 between two UEs, the achieved signal-to-interference ratio (SIR) will be only around $20 \mathrm{~dB}$ for each UE when their information are decoded at the base station (BS) with a simple maximum ratio combining.

\section{B. Challenges}

Challenge 1: precise characterization of massive MIMO channels. A simple one-ring model is widely used in outdoor

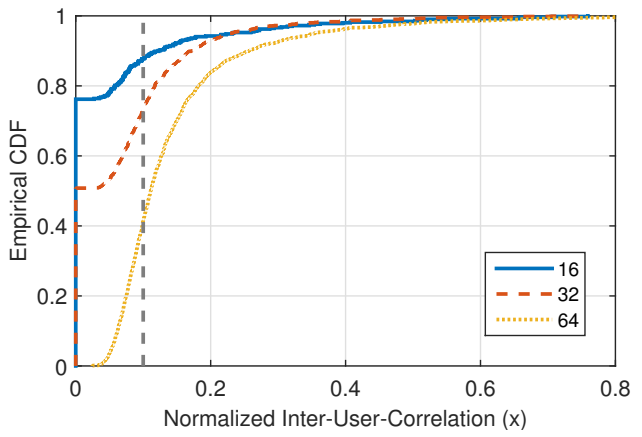

Fig. 1: The high user density leads to a significant IUC. In a dense scenario, the system performance should be benefited from a proper user selection. The results are obtained from our measurements with the KU Leuven distributed massive MIMO testbed that will be detailed in Sec. III.

scenarios to characterize massive MIMO channels [2]. It reflects that a BS is surrounded by a limited number of scatterers. However, from real outdoor measurements, especially in the non-line-of-sight (NLoS) scenarios [3,4], the angular information is very complicated as the impinging signal and reflectors to the array elements are from multiple randomly positioned scatterers. On the other hand, in indoor, even in line-ofsight (LoS) scenarios, when there are many reflectors in the environment, the one-ring model is insufficient for the system evaluation. The extended COST 2100 model as proposed in [5] is adjusted from massive MIMO channel measurement campaign, however, the extension and some parameters are obtained from a virtual linear array. The mutual coupling which causes gain variation [6] among the finite antennas is not considered in a virtual antenna array. Moreover, the mutual coupling, which impacts the detection of the cluster visibility region from angular transform algorithms [7], can also hardly be exhibited in the virtual array. To date, no comprehensive model can precisely characterize the channel of large-scale antenna arrays. Therefore, in this paper, we design and evaluate system algorithms based on channel measurements of a real propagation environment.

Challenge 2: efficient user selection in indoor dense massive MIMO networks. When users are closely located, a single BS might not be able to separate the users as for instance already their angular information is shared. Distributed arrays can help to better serve closely located users, at the cost of power imbalances caused by different path-losses between the users and the distributed array elements. However, in dense massive MIMO networks, a better strategy could be to schedule UEs 
by taking into account the IUC and improve user separation by user scheduling. In [8], channel state information (CSI) from all UEs in the one-ring model is assumed to be known beforehand. The idea is to group UEs by the largest chordal distance. Its key drawback is that it builds a comprehensive relationship of all UEs, which is quite computational demanding and impractical in a dynamic environment. Another point that should not be ignored is the power difference among these UEs. Most of the previous work have analyzed the channel after normalizing all UEs to be with equal received power. The equal power assumption is reasonable when power control is applied and the boosted gain can compensate the large scale fading plus shadowing, but the headroom for power control is not always sufficient to bridge the gap.

\section{Contributions}

We design a low complexity scheduling algorithm to recoup the performance of dense massive MIMO. We summarize our contributions as below.

Contribution 1. By applying 10m optical fibers to link the main computation engine with the remote radio heads (RRHs), in our massive MIMO testbed, we distribute the 64 antennas from one collocated array to two sub-arrays. Besides, we propose a method to compensate the extra delay caused by the longer cables. (Sec. III)

Contribution 2. Channel measurement in the indoor conference room with 64 UEs was conducted with four different ways of BS antenna deployments. This enables us to further exploit the combination of user scheduling (US) algorithm and the antenna arrangement in the environment. (Sec. III and V)

Contribution 3. A sub-optimal low complexity greedy US algorithm is designed and evaluated with real channel measurements. The algorithm can increase the system sum rate by up to $60 \%$ and by $30 \%$ when averaged out in four different scenarios. (Sec. IV and V)

\section{SySTEM DESCRIPTION}

\section{A. Problem formulation}

We consider a dense network as shown in Fig. 2. The BS is equipped with $M$ antennas and there are $S$ single antenna UEs. $\mathcal{S} \triangleq\{1, \ldots, S\}$ represents the set of all the UEs. For the US, the BS at the start of any coherence time interval $\tau_{c}$, selects $K$ out of $S$ UEs for multi-user MIMO data transmission. In this paper, we assume the CSI is known beforehand. It is also assumed that in the time division duplexing (TDD) based system, the coherence time is a summation of both UL and down-link (DL) data transmission time, $\tau_{c}=\tau_{u}+\tau_{d}$.

\section{B. Physical channel representation}

The frequency response of CSI, $\mathbf{h}_{k}(q) \in \mathbb{C}^{M}$ from UE $k$ at subcarrier $q$ is represented as

$$
\mathbf{h}_{k, q}=\mathbf{h}_{k}\left(f_{c}+q \Delta f\right)=\beta_{k}^{\frac{1}{2}} \mathbf{g}_{k, q},-\frac{Q}{2} \leq q \leq \frac{Q}{2},
$$

where $\beta$ denotes the large scale fading, $f_{c}$ is the center frequency, $\Delta f$ is the subcarrier spacing and $Q$ is the number

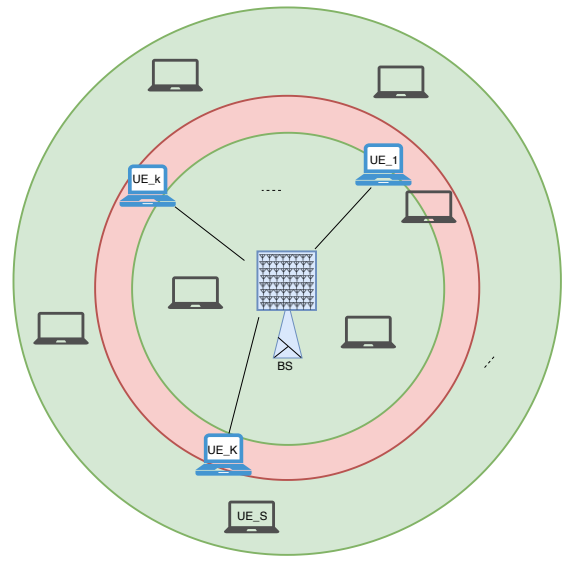

Fig. 2: The problem of user scheduling in a massive MIMO system. The goal is to select $K$ UEs among a total of $S$ UEs. While maximizing the spectral efficiency, the user fairness is another crucial factor to be considered.

of subcarriers. The small scale fading vector has a fixed sum power $\operatorname{tr}\left(\mathbb{E}\left\{\mathbf{g}_{k, q} \mathbf{g}_{k, q}^{H}\right\}\right)=M$.

\section{UL and DL Spectral Efficiency}

The analysis of the spectral efficiency (SE) provides us key information in designing the US algorithms. Without loss of generality, we drop off the subcarrier index $q$ unless specified. Moreover, due to the channel hardening in massive MIMO [2], frequency selective fading is averaged out. Therefore, the same selected user set is applied to all subcarriers in a coherence block.

The US algorithm is to select a set of active UEs, denoted by $\mathcal{S}_{a c}$, given that $\mathcal{S}_{a c} \subset \mathcal{S}$ and $\left|\mathcal{S}_{a c}\right|=K$. Herein, the UL received signal at the BS from the selected $K$ active UEs can be represented as:

$$
\mathbf{y}=\sum_{k \in \mathcal{S}_{a c}} \mathbf{h}_{k} s_{k}+\mathbf{n},
$$

where $\mathbf{y} \in \mathbb{C}^{M}$, the complex Gaussian noise is independent and identically distributed (i.i.d.) $\mathbf{n} \sim \mathcal{N}_{\mathbb{C}}\left(\mathbf{0}_{M}, \sigma_{U L}^{2} \mathbf{I}_{M}\right)$. The UL signal from UE $k$ is denoted by $s_{k} \in \mathbb{C}$ and has power $p_{k}=\mathbb{E}\left\{\left|s_{k}\right|^{2}\right\}$. The BS calculates a spatial discrimination vector $\mathbf{v}_{k}$ to decode data stream from UE $k$. The minimum mean square error (MMSE) combiner maximizes the signal to interference noise ratio (SINR) and is represented as [2]

$$
\mathbf{v}_{k}=p_{k}\left(\sum_{i \in \mathcal{S}_{a c}} p_{i}\left(\mathbf{h}_{i} \mathbf{h}_{i}^{H}\right)+\sigma_{U L}^{2} \mathbf{I}_{M}\right)^{-1} \mathbf{h}_{k} .
$$

If we define a diagonal matrix $\mathbf{P}=\operatorname{diag}\left(p_{1}, \ldots, p_{K}\right)$, the combining vectors for all UEs after some matrix transformation, can be collected and be represented in a compact form,

$$
\mathbf{V}=\left[\mathbf{v}_{1} \ldots \mathbf{v}_{K}\right]=\mathbf{H}\left(\mathbf{H}^{H} \mathbf{H}+\sigma_{U L}^{2} \mathbf{P}^{-1}\right)^{-1},
$$

where the channel matrix $\mathbf{H}=\left[\mathbf{h}_{1} \ldots \mathbf{h}_{K}\right]$. The resulted SINR is therefore $[2,9]$

$$
\operatorname{SINR}_{k}^{\mathrm{UL}}=\frac{\rho_{k}}{\sigma_{U L}^{2}\left[\left(\mathbf{H}^{H} \mathbf{H}+\sigma_{U L}^{2} \mathbf{P}^{-1}\right)^{-1}\right]_{k k}}-1 .
$$




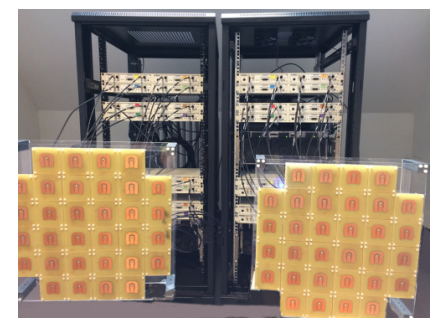

(a) KUL Massive MIMO testbed.

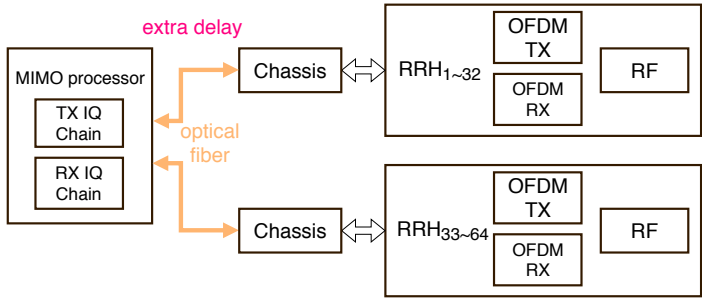

(b) Block diagram.

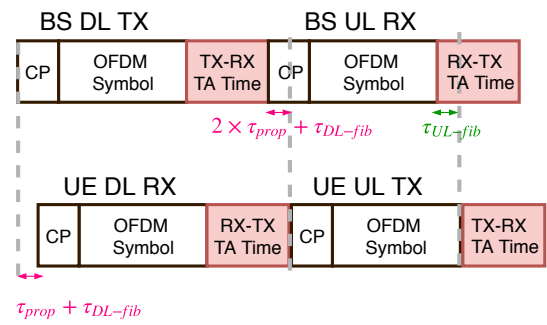

(c) Compensation of the extra delay.

Fig. 3: Our distributed massive MIMO testbed in KU Leuven. It consists of two distributable 32-antenna arrays. The two sub-systems, each with 32 RRHs, are connected by two 10 meters optical fibers as back-haul connections to the central MIMO processing unit.

Finally, the average UL or DL SE $S E_{s}$ per UE is defined as

$$
S E_{s}=\frac{\tau_{s}}{\tau_{c}} \mathbb{E}\left(\log _{2}\left(1+\operatorname{SINR}_{s}\right)\right),
$$

where $s=u$ or $d$, for UL or DL, respectively ${ }^{1}$. All expectations are with respect to the channel realizations.

From Eq. (5) and [10], we observe that a low average channel gain variance in the selected UEs set is highly preferable. The average channel gain per UE can be represented as

$$
\overline{\mathbf{h}}_{k}=\mathbb{E}\left\{\left\|\mathbf{h}_{k}(q)\right\|^{2}\right\} .
$$

\section{Testbed Measurement}

Most of the measurement campaigns and testbed design focus on the collocated antenna array topology [10]-[13]. Our massive MIMO testbed in KU Leuven is designed for distributed massive MIMO research [14,15]. In this section, we first introduce how we can run two distributed arrays in our system. Then, we describe the conducted indoor channel measurement in a conference room.

\section{A. Distributed massive MIMO testbed}

Our distributed massive MIMO testbed in KU Leuven, as shown in Fig. 3a, consists of 32 National Instruments universal software radio peripherals (USRPs). The 32 USRPs are equally divided into two sub-systems and each of them supports 32 RRHs. The two sub-systems are connected to the MIMO processor as shown in Fig. 3b via two 10 meters optical fibers responsible for the back-haul In-phase and quadrature components (I/Q) centralized processing. Note that since one of the sub-systems is bundled to the MIMO processor in the same rack, so the maximum separation of the two subsystems can be $10 \mathrm{~m}$ instead of $20 \mathrm{~m}$. With optical fibers, some extra fiber delays, i.e., $\tau_{D L-f i b}$ and $\tau_{U L-f i b}$ (DL and UL fiber delay, respectively) are generated as compared to the short Multisystem eXtension Interface (MXI) cooper cable ${ }^{2}$ assembled in the collocated system [11].

\footnotetext{
${ }^{1}$ In the simulation section, we omit the pre-log factor.

${ }^{2}$ With fiber, the propagation speed is approximately $80 \%$ as compared to a copper. In our case, the delayis around 7 OFDM samples. We explain this to point out that distributing the system implies some extra testing and development. In the system design of this work, the antennas are distributed in a sub-system manner. In [14], we applied long SubMiniature version A (SMA) cables in the output port of the RF to the antenna. The introduced propagation delay differs from the one in this paper. Also, there are extra propagation loss in the previous design, though it is capable of being more distributed in the per RRH manner.
}

The extra delay can be tolerated in our TDD based massive MIMO system. This can be understood from an example frame structure shown in Fig. 3c. Keep in mind that the frame timing is managed by the BS. To begin with, in the DL, the OFDM symbol reaches the UE with wireless propagation delay $\tau_{\text {prop }}$ plus the $\tau_{D L-f i b}$. Then, the UE starts a TX OFDM symbol after a RX-TX turnaround (TA) time counter. The BS starts the receive mode by assuming the UL symbol reaches the BS after a TX-RX TA time. As we can see from the flow, the two times of $\tau_{\text {prop }}$ and the $\tau_{D L-f i b}$ can be covered by the cyclic prefix (CP) in the UL symbol ${ }^{3}$. The UL I/Qs reach the MIMO processor in the BS with $\tau_{U L-f i b}$, which can be covered by a proper design of the RX-TX TA time in the BS. Note that for a distributed massive MIMO system, other design perspectives have to be considered. For instance, the TDD reciprocity calibration algorithm of a distributed massive MIMO has been improved in [15], from the viewpoint that it reduces the DL precoded inter user interference (IUI).

\section{B. Indoor channel measurement}

The indoor channel measurement was conducted in a meeting room with size of $13.2 \mathrm{~m} \times 7.1 \mathrm{~m}$ as shown in Fig. 4 with two distributed antenna arrays labeled as $A 1$ and $A 2$ for array one and array two, respectively. Each of the array is equipped with 32 patch antennas. A combination of four different deployments in the massive MIMO BS are collected: 1) $\mathrm{Cl}$ stands for the scenario Collocated 1 , where $\mathrm{A} 1$ and $\mathrm{A} 2$ are connected in the front of the meeting room. 2) $C 2$ stands for the scenario Collocated 2, where A1 and A2, next to each other, are set aside of the meeting room with $30^{\circ}$ clockwise rotation from the front side wall. 3) $\mathrm{Dl}$ stands for the scenario Distributed 1, where A1 and A2 are set in both corners of the front of the room. They are also with $30^{\circ}$ rotation, one clockwise and the other counter clockwise from the wall. 4) $D 2$ represents the scenario Distributed 2, where A1 is located in the front of the room, and A2 was set in the side of the room with $45^{\circ}$ clockwise rotation. The inter-array distance of $\mathrm{A} 1$ and $\mathrm{A} 2$ is about $7 \mathrm{~m}$.

During the measurement, the environment was kept static. Nevertheless, each measurement on the UE side is collected one by one by moving two USRPs, where each USRP acts as two UEs. Every two UEs on the same USRP have a distance

\footnotetext{
${ }^{3}$ It can also be compensated by shortening the TX-RX TA time in the BS.
} 


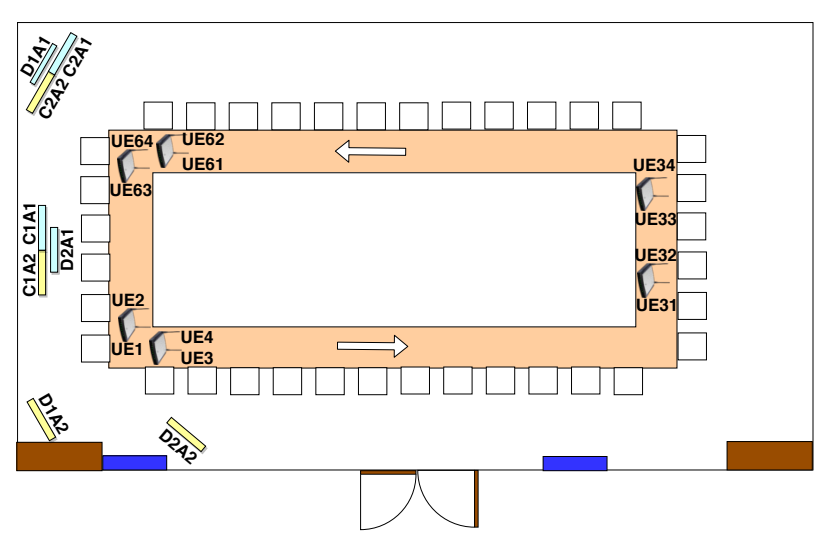

(a) Block diagram of the measurement setup.

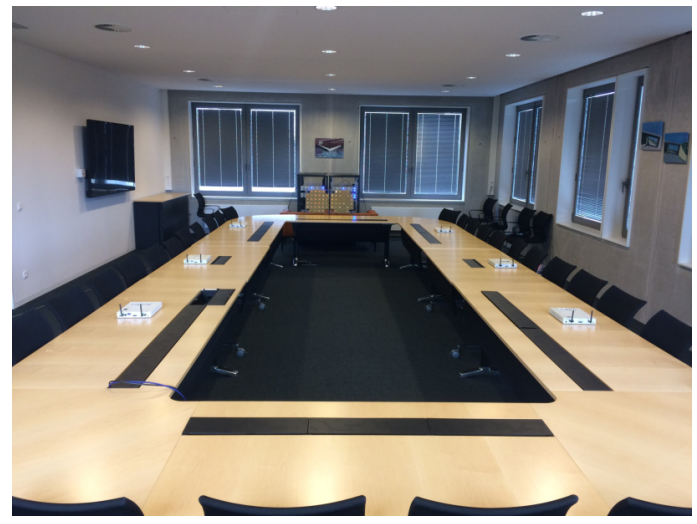

(b) Snapshot of the measurement setup.

Fig. 4: The indoor channel measurement is conducted in a meeting room with four different antenna array deployments: C1, C2, D1, and D2 (C: collocated scenarios; D: distributed scenarios). We measure channels from 64 UEs for the four different deployment of antenna arrays.

of around two wavelengths. As indicated in Fig. 4a, we deploy only four UEs at each time instant. As represented by the arrow in the figure, the two USRPs are moved step-wise towards the end of the table opposite to the antenna array and turned around towards the array for a total of 16 locations to emulate 64 UEs.

At each UE location, a total of 80 OFDM UL pilot symbols are collected, where the OFDM frame is LTE-like radio frame structure. With a frequency-division multiplexing of the pilot symbol, each UE occupies 100 subcarriers with 12-subcarrier spacing. The sampling rate is $30.72 \mathrm{MHz}$ and the system is running at $2.61 \mathrm{GHz}$. The detail design of our patched antenna can be referred to [6].

\section{Proposed User Scheduling Algorithm}

Due to the UL-DL duality [16], we focus on US in the UL, applied to both links. Decision made from only UL CSI is particularly suited to a TDD based system. From the system performance perspective, selecting a subset of UEs with the lowest IUI is always preferable. However, selection is complex, as according to (4), the combining matrix with a matrix inversion is mutually dependent on the UEs in the selected set. Moreover, finding the optimal US, typically requires an exhaustive performance evaluation of all combinations, incurring prohibitively high computation cost even with a moderate number of UEs. Thus, we apply the main concept in a suboptimal greedy US approach $[17,18]$, but with a modified per iteration UE appending algorithm.

We detail the proposed scheduling algorithm in Algorithm 1. The main concepts are introduced as follows. In each scheduled resource block, for fairness, we pick the first UE with the lowest historical SE.

$$
k_{1}=\underset{k \in \mathcal{S}}{\arg \min } S E_{k} .
$$

Next, decisions are made about which users to select in the consecutive iterations. In summary, each of the $i_{t h}$ iteration is decomposed into four steps. 1) For the searching of each $i_{t h}$ UE, we define an infinite IUC threshold $\delta_{\max }$ (Line 3 in Algorithm 1). 2) The proposed US APPEND $_{\text {algorithm which we }}$ will explain later calculates an IUC set $\Delta_{i}$. The set contains the inter-user correlations between the new UE under examination to all the previous $i-1$ UEs that have been determined. The $i_{\text {th }} \mathrm{UE}$ is a potential candidate in this iteration if the maximum in the set $\Delta_{i}$ is lower than the threshold $\delta_{\max }$. (Line 4-7 in Algorithm 1). When the criterion is met, a temporarily set with superscript "New" is updated. Meanwhile, the threshold is also updated to the new lower number (Line 8-11). It is worth mentioning that we evaluate all the $\left|\mathcal{S}_{u e}\right|$ UEs in each $i_{t h}$ iteration. 3) We update the final set after checking all the possibilities (Line 14). 4) The whole US process is finished when all the $K$ UEs are found. Afterwards, the new averaged SE per UE is updated (Line 16). Note that there is no special requirement to set the initial values or threshold. The algorithm can be applied to any scenario.

Now, we introduce the proposed methods for the $\mathrm{US}_{\mathrm{APPEND}}$ algorithm. The proposed method is called Gram-matrix based US (GMUS), which is a very low complexity US method that can minimize the IUC.

\section{A. Proposed Gram Matrix based User Selection (GMUS)}

Some insights about a two-UE scenario has been studied in [10]. Two vital findings from the study can be considered when appending the $i_{t h}$ UE in every iteration. This can be observed by the Gram matrix $\mathbf{C}_{i}$ associated with $\mathbf{H}_{i}=\left[\mathbf{h}_{k_{1}} \ldots \mathbf{h}_{i}\right]$ (Note that at iteration $i$, a number of $i-1$ UEs have been decided):

$$
\mathbf{C}_{i}=\mathbb{E}\left(\mathbf{H}_{i}^{H} \mathbf{H}_{i}\right)=\left[\begin{array}{cc}
\mathbf{C}_{i-1} & \mathbf{c}_{i} \\
\mathbf{c}_{i}^{H} & c_{i, i}
\end{array}\right]
$$

When the $i_{t h}$ UE is appended, $i-1$ elements of new IUCs, i.e., $\mathbf{c}_{i}$ is generated. The first reason for the bad SINR comes from the gain imbalance between the UEs, which we can observe from the diagonal terms. The second factor is the IUC terms which can be interpreted from the non-diagonal upper triangular terms. Intuitively, we can first limit the power difference between the candidate UE and selected UEs, then minimize their IUC. However, in a real scenario, when the UEs share similar power, they might locate closely with a high probability. With this limitation, we might miss the best 


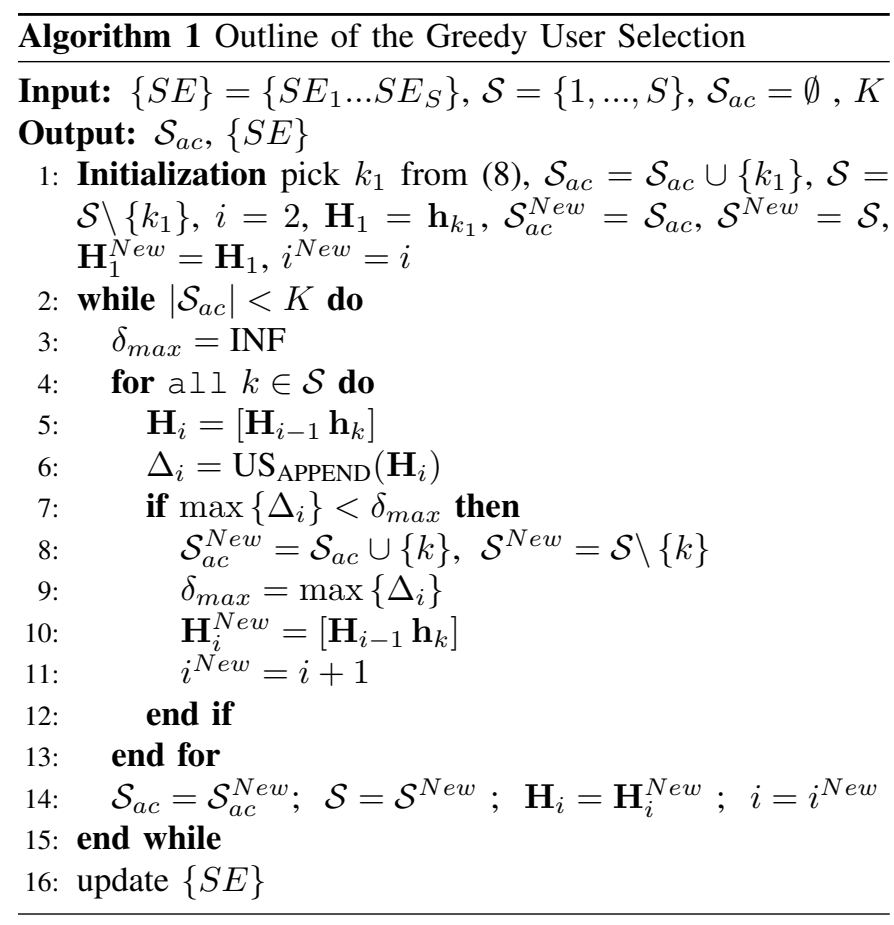

option. Thus, here we treat it differently. At each iteration $i$, we normalize the new generated IUC $\mathbf{c}_{i}$ with respect to $c_{i, i}$ to build the new IUC set $\Delta_{i}$ :

$$
\Delta_{i}=\hat{\mathbf{c}}_{i}=\left|\mathbf{c}_{i}\right| \circ\left(c_{i, i}\right)^{-1} .
$$

The reason for this normalization is to avoid the amplitude of the incoming scheduled UE to bias the decision. The target is to minimize the maximum of $\Delta_{i}$ as shown in Line 7 of Algorithm 1, for all the remaining candidates in the $\mathcal{S}$.

\section{Performance Evaluation}

Before introducing the system level simulation, we first give an overview of the power received from the channel measurements. In any scenario, the BS does not receive same power from all $\mathrm{UEs}^{4}$. The averaged channel gain per UE as defined in (7) to demonstrate the gain variation of the UEs in the four scenarios is plotted in Fig. 5. Notably, the received power from the two arrays A1 and A2 is separately plotted for comparison. The gain distribution shows the following two main features, namely, array closeness and array directivity. In array closeness, closer UEs to the BS usually benefit from higher desired signal strength. In the collocated scenarios, two

\footnotetext{
${ }^{4}$ Even if we put the array in the center of the table, due to the directivity of the array, the asymmetry in the receive signal power cannot be alleviated.

TABLE I: System parameter settings in the evaluation.

\begin{tabular}{|l|l|}
\hline$\rho_{k}$ & 1 \\
\hline SNR & $20 \mathrm{~dB}$ \\
\hline US routine & 1000 \\
\hline Number of subcarriers & 100 \\
\hline Number of OFDM symbols & 80 \\
\hline$K$ & 10 \\
\hline$|\mathcal{S}|$ & 16,32, and 64 \\
\hline $\mathrm{M}$ & 64 \\
\hline Scenarios & $\mathrm{C} 1, \mathrm{C} 2, \mathrm{D} 1$ and D2 \\
\hline
\end{tabular}
}
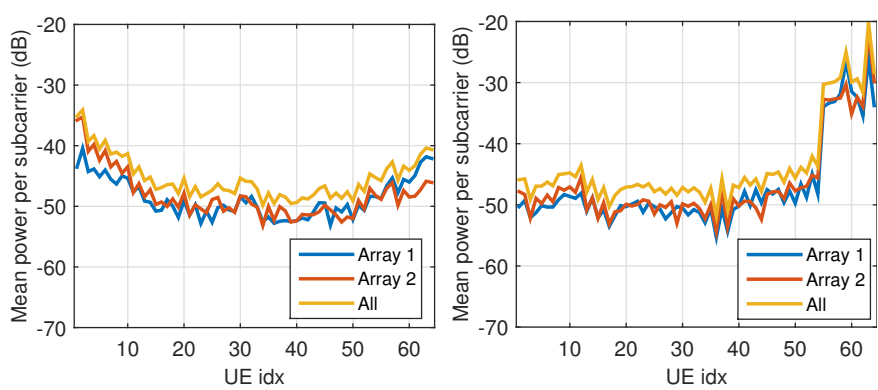

(a) User power distribution in $\mathrm{C} 1$.

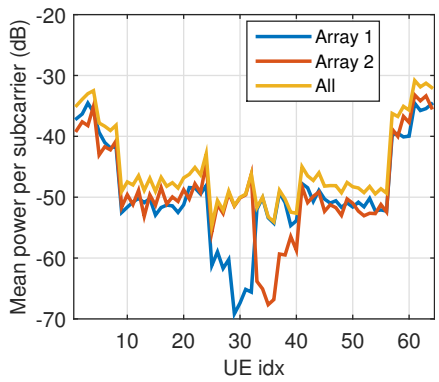

(b) User power distribution in $\mathrm{C} 2$

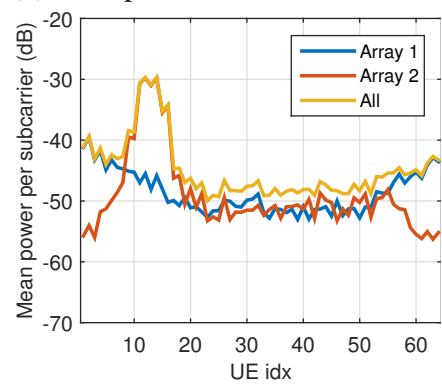

(c) User power distribution in D1. (d) User power distribution in D2.

Fig. 5: Averaged sum power of the number of antennas over all subcarriers and time for each UE position.

arrays receive a similar power level from the UEs; while in the distributed scenarios, there is gain imbalance between the two arrays. So, it is easier to calculate optimum power control per UE in the collocated scenarios. However, locating arrays at similar locations could imply higher CSI correlation at the BS. The joint contribution from both gain imbalance and channel correlation is given in the US results. In array directivity, the direction of the array's main beam impacts even more than the distance. Fig. 5b reveals that UEs with indices 58-64 receive approximately $15 \mathrm{~dB}$ higher power than their neighbors. The cross-reference to our measured array pattern in an anechoic chamber as in Fig. 9 [6] indicates that the gain difference between the main beam and the side lobes is more than 12 dB. Fig. 5b shows higher deviation, which can be explained by extra contribution from the environment reflection.

The performance of US algorithms is evaluated by a system level simulation with the conducted measurements. The average throughput in each scenario is numerically evaluated by summing the averaged throughput of the $K$ UEs over 1000 scheduled routines. After each US routine, the averaged historical SE is updated, which affects the first UE being selected in the next routine. The channel is assumed to be quasi-static, and the BS has knowledge about CSI from all UEs in advance ${ }^{5}$. The signal-to-noise ratio (SNR) of the simulation refers to the average single antenna SNR as perceived by a single UE with the maximum received power in that scenario, $\mathrm{SNR} \triangleq \frac{\max \left(\mathbb{E}\left(\overline{\mathbf{h}}_{k}\right)\right)}{M \sigma_{U L}^{2}}$. The parameters of the system level simulation are listed in Table I. Specifically, the averaged sum rate of the proposed greedy US algorithm with the GMUS is

\footnotetext{
${ }^{5}$ Note that in a static environment of a TDD based massive MIMO, the BS is able to collect CSI from all UE after several slot of UL channel training.
} 


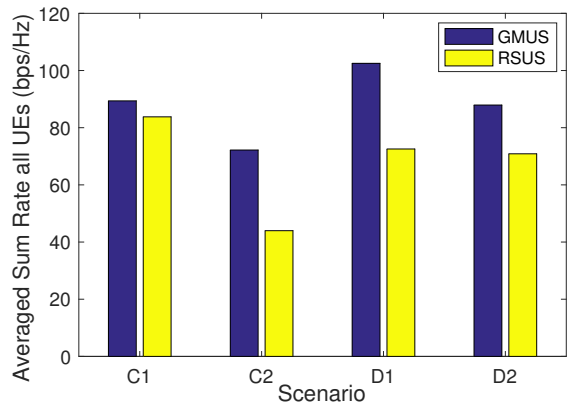

(a) Select 10 UEs out of total 64 UEs

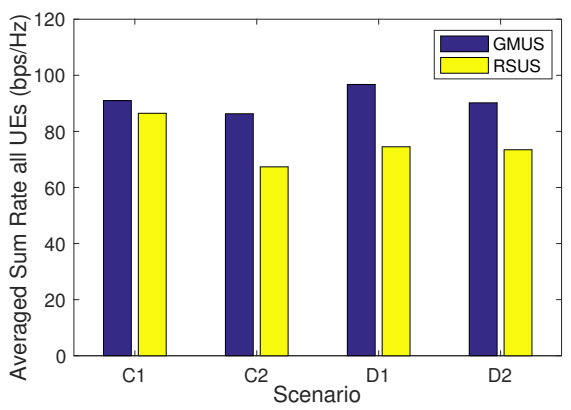

(b) Select 10 UEs out of total 32 UEs

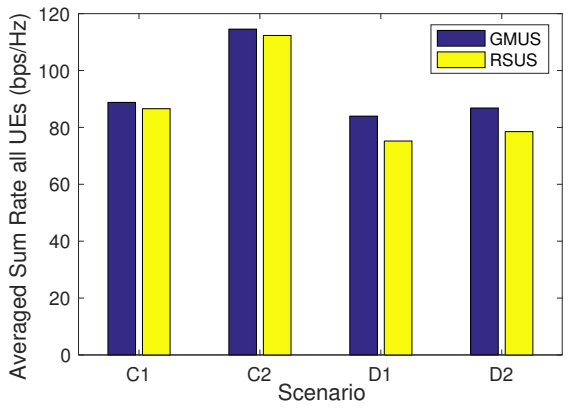

(c) Select 10 UEs out of total 16 UEs

Fig. 6: Comparison of the UL averaged sum rate for the combination of the three criterion and four antenna topology. We compare the overall 64 UEs to the case when the UEs are sub-sampled to a sparse distance. The fewer the number of total UEs, the sparser their distance.

compared with a random selection US (RSUS). The results are shown in Fig. 6. In RSUS, $K$ out of $|\mathcal{S}|$ are randomly selected. In the case where 10 UEs are selected among the 64 , the proposed method outperforms RSUS by more than $60 \%$ in the scenario $\mathrm{C} 2$ and it improves by $30 \%$ when averaged out in four different scenarios. Moreover, with a good US method, the distributed scenarios are better than collocated scenarios for more than $18 \%$ on average. The result indicates that potentially we have more UEs which share less channel correlation in the distributed arrays than the collocated arrays.

We further do a sub-sampling of the UE in the conference room to evaluate the impact of densely located UEs. For one comparison scenario, we choose one UE over every two UEs and get an overall of 32 UEs as shown in Fig. 6b. Likewise, one among every four UEs are sub-sampled, the result is plotted in Fig. 6c. Interestingly, the performance gap between the two methods in the $\mathrm{C} 1$ is always less than those of the other three scenarios, implying that there is a less extreme high or low correlation between the UEs. Another observation is that the above-average sum rate of the proposed GMUS reduces when the UEs do not deploy densely in the environment. It reveals that in the future, in a dense network, a smart US combined with distributed arrays has a high potential to be considered in the advanced system-level design.

\section{CONCLUSION}

In this paper, we aim to tackle a potential issue rising in the massive MIMO empowered dense networks. Due to the closely located users in many scenarios like a conference room or a coffee shop, the high inter-user correlation causes a significant burden for the system throughput. Physically, we can distribute the arrays to lower down the user correlation. The distribution of the arrays can also combine with higher layer user scheduling algorithm. In the measured real propagation scenario, we demonstrated that the combination of the distributed arrays with our proposed Gram-matrix based greedy user selection algorithm outperforms the collocated scenario with the same method for more than $18 \%$ on average. For the future work, the trade-off between the improvement in system throughput and user fairness is an interesting topic.

\section{REFERENCES}

[1] T. L. Marzetta, "Noncooperative cellular wireless with unlimited numbers of base station antennas," IEEE Transactions on Wireless Communications, 2010.

[2] E. Björnson, J. Hoydis, L. Sanguinetti et al., "Massive mimo networks:

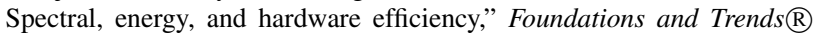
in Signal Processing, 2017.

[3] X. Gao, O. Edfors, F. Rusek, and F. Tufvesson, "Massive mimo performance evaluation based on measured propagation data." IEEE Trans. Wireless Communications, 2015.

[4] C.-M. Chen, A. P. Guevara, and S. Pollin, "Pilot contamination in massive mimo: A measurement-based analysis using 2d-music," IEEE Signal Processing Advances in Wireless Communications, 2018.

[5] X. Gao, J. Flordelis, G. Dahman, F. Tufvesson, and O. Edfors, "Massive mimo channel modeling-extension of the cost 2100 model," in Joint NEWCOM/COST Workshop on Wireless Communications, 2015.

[6] C.-M. Chen, V. Volski, L. Van der Perre, G. Vandenbosch, and S. Pollin, "Finite large antenna arrays for massive mimo: characterisation and system impact," IEEE Transactions on Antennas and Propagation, 2017.

[7] B. Friedlander and A. Weiss, "Direction finding in the presence of mutual coupling," IEEE trans. on antennas and propagation, 1991.

[8] Y. Xu, G. Yue, and S. Mao, "User grouping for massive mimo in fdd systems: New design methods and analysis," IEEE Access, 2014.

[9] Y. Jiang, M. K. Varanasi, and J. Li, "Performance analysis of zf and mmse equalizers for mimo systems: An in-depth study of the high snr regime," IEEE Transactions on Information Theory, 2011.

[10] X. Gao, O. Edfors, F. Rusek, and F. Tufvesson, "Linear pre-coding performance in measured very-large mimo channels." in VTC Fall, 2011.

[11] J. Vieira, S. Malkowsky, K. Nieman, Z. Miers, N. Kundargi, L. Liu, I. Wong, V. Öwall, O. Edfors, and F. Tufvesson, "A flexible 100-antenna testbed for massive mimo," in Proc. Globecom Workshops, 2014.

[12] S. Payami and F. Tufvesson, "Channel measurements and analysis for very large array systems at $2.6 \mathrm{ghz}$," in Proc. European Conference on Antennas and Propagation, 2012

[13] M. Gauger, J. Hoydis, C. Hoek, H. Schlesinger, A. Pascht, and S. ten Brink, "Channel measurements with different antenna array geometries for massive mimo systems," in Proc. ITG SCC, 2015.

[14] C.-M. Chen, V. Volskiy, A. Chiumento, L. Van der Perre, G. A. Vandenbosch, and S. Pollin, "Exploration of user separation capabilities by distributed large antenna arrays," in Globecom Workshops, 2016.

[15] C.-M. Chen, S. Blandino, A. Gaber, C. Desset, A. Bourdoux, L. Van der Perre, and S. Pollin, "Distributed massive mimo: A diversity combining method for tdd reciprocity calibration," in IEEE GLOBECOM, 2017.

[16] M. Schubert and H. Boche, "Solution of the multiuser downlink beamforming problem with individual sinr constraints," IEEE Transactions on Vehicular Technology, 2004.

[17] G. Dimic and N. D. Sidiropoulos, "On downlink beamforming with greedy user selection: performance analysis and a simple new algorithm," IEEE Transactions on Signal processing, 2005.

[18] D. Hammarwall, M. Bengtsson, and B. Ottersten, "Utilizing the spatial information provided by channel norm feedback in sdma systems," IEEE Transactions on Signal Processing, 2008. 